\title{
250 Jahre
}

\section{rechtswissenschaftlicher}

Verlag C.H.Beck

$$
1763-2013
$$

\section{von}

Lwe Wesel 


\section{Jahre \\ rechtswissenschaftlicher \\ Verlag C.H.Beck}

$1763-2013$ 
https://doi.org/10.17104/9783406684883-

Generiert durch IP '172.22.53.54', am 26.04.2023, 11:13:15.

Das Erstellen und Weitergeben von Kopien dieses PDFs ist nicht zulässig. 


\section{Jahre \\ rechtswissenschaftlicher \\ Verlag C.H.Beck}

$$
1763-2013
$$

von

Uwe Wesel

und

Hans Dieter Beck

sowie Mitarbeitern des

Verlages C.H.Beck 


\section{www.beck.de}

ISBN 9783406656347

(C) 2013 Verlag C.H.BECK oHG

Wilhelmstraße 9, 8o801 München

Druck und Bindung: CPI - Ebner \& Spiegel GmbH

Eberhard-Finckh-Straße 61, 89075 Ulm

Satz: Fotosatz Amann, Aichstetten

Gestaltung: Konstanze Berner, München

Grafiken: Jörg Schäffer, München

Kartographie: Ulrich Grebe, Essen

Gedruckt auf säurefreiem, alterungsbeständigem Papier

(hergestellt aus chlorfrei gebleichtem Zellstoff) 


\section{Vorwort}

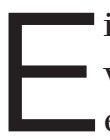

in Verlagsunternehmen, 250 Jahre im Besitz und erfolgreich geführt von einer Verlegerfamilie, ist eine Seltenheit, die angemessen gefeiert werden muss. Dazu gehört auch eine Jubiläumsschrift; sie soll die wechselvolle Geschichte des Verlages C.H.Beck aus heutiger Sicht nachzeichnen.

Dabei waren die Rechtswissenschaft und die sonstigen geisteswissenschaftlichen Bereiche nebst Belletristik in zwei getrennten Werken zu behandeln. Die Stofffülle wäre sonst zu groß geworden.

Nun hat unser Verlag im Jahr 2007 eine Festschrift mit dem Titel «Rechtswissenschaft und Rechtsliteratur im 2o. Jahrhundert» von Dietmar Willoweit und anderen Autoren erscheinen lassen. Darin wurden 100 Jahre Rechtsliteratur - insbesondere von C.H.Beck - im Zusammenhang mit der rechtswissenschaftlichen Entwicklung von namhaften Wissenschaftlern nach Sachgebieten geordnet beschrieben. Musste nun zum 250-jährigen Gründungstag erneut eine Festschrift für den juristischen Verlag erscheinen? Die Antwort lautete «ja»; denn das Interesse liegt bei einem solchen Jubiläum nicht nur auf den letzten hundert Jahren, sondern auf der gesamten Zeit von 1763 bis heute. Und es stehen bei der neuen Jubiläumsschrift nicht nur die Werke und die Autoren im Mittelpunkt, sondern das gesamte Unternehmen C.H.Beck mit seinem gesellschaftlichen Umfeld und mit seinen wirtschaftlichen Bedingungen.

Dieses Buch erscheint in einer Zeit rapider Wandlung. Das Interesse der Juristen wendet sich den Datenbanken und den Informationen aus dem Internet $\mathrm{zu}$ und ob die Rechtsliteratur in ihren bisherigen gedruckten Formen dominant bleiben wird, erscheint für die Zukunft jäh in Frage gestellt. Umso mehr macht es Sinn, den bis jetzt erreichten Stand der Literatur im Beck Verlag aufzuzueigen.

Diese Jubiläumsschrift wäre kaum vorstellbar, hätten wir nicht dafür den in der deutschen Rechtsgeschichte umfassend erfahrenen und anerkannten Rechtshistoriker Uwe Wesel gewinnen können. Er ist mit dem Beck Verlag durch seine «Geschichte des Rechts» sowie seine «Geschichte des Rechts in Europa» eng verbunden. Es war für ihn als einzigen Autor 
eine nicht mehr erfüllbare Aufgabe, den langen historischen Zeitraum von 250 Jahren zu erschließen und zugleich der großen Fülle der Werke aus der neueren Zeit gerecht zu werden. Deshalb haben Verlagslektoren bei der letzten Phase der Manuskripterstellung mitgewirkt und haben neuere Sachgebiete und Verlagswerke beschrieben. Den deutlich größten Teil, nämlich die Zeit von 1763 bis 1970, hat Uwe Wesel geschrieben. Für die Zeit danach haben - in unterschiedlichem Umfang - folgende Verlagslektoren mitgeholfen:

Anna von Bonhorst, Albert Bucholz, Dieter Burneleit, Dr. Wolfgang Czerny, Dr. Christiane Dobring, Gerald Fischer, Dr. Adrian Hans, Andreas Harm, Dr. Heino Herrmann, Matthias Hoffmann, Dr. Roland Klaes, Dr. Wolfgang Lent, Professor Dr. Klaus Letzgus, Thomas Marx, Andreas Mirbt, Dr. Rolf Müller, Philipp Mützel, Dr. Thomas Schäfer, Ruth Schrödl, Professor Dr. Achim Schunder, Stefan Tischler, Dr. Wilhelm Warth, Dr. Johannes Wasmuth, Professor Dr. Klaus Weber und Dr. Klaus Winkler.

Da für die Arbeit dieser Verlagsmitarbeiter nicht mehr viel Zeit zur Verfügung stand, muss für manche Unterlassung und für nicht optimale Verteilung der Schwerpunkte sowie die eine oder andere Ungenauigkeit um Nachsicht gebeten werden. Auch hat jeder, der am Manuskript mitgewirkt hat, einen etwas verschiedenen Formulierungsstil. Nichtsdestoweniger haben Professor Wesel und wir uns bemüht, dem Werk eine harmonische Gesamtform zu geben.

Der Verleger hat am Schluss die Entwicklung von beck-online beschrieben, hat Anmerkungen zum Gesamtunternehmen C.H.Beck gemacht und einen Ausblick in die Zukunft zu geben versucht.

München, im September 2013

Dr. Hans Dieter Beck 


\section{Inhalt}

I. Einleitung. Sechs Generationen in vier Jahrhunderten 17

II. Konturen des Anfangs 19

1. Nördlingen 19

2. Vier Kriege und ihre Folgen 21

3. Veränderungen im Buchhandel 26

III. Carl Gottlob, Luise, Carl Heinrich und Catharina Magdalena Beck. 1763-1846. Die Ersten 31

IV. Märzrevolution und Reichsgründung. 1848-1871 43

V. Carl und Eugenie Beck, Ernst Rohmer als Statthalter für die Söhne Carl Becks. 1846-1884. Intensivierung des Juristischen 47

VI. Kaiserreich, Weimarer Republik und Buchhandel. 1871-1933 57

VII. Oscar Beck. 1884-1924. Mit dem Umzug nach München auch juristisch etwas weiter nach oben 65

VIII. Schwabing. Arbeiter, Studenten, Literaten und Künstler 77

IX. Heinrich Beck 1924-1933. Bedeutungsverlust im Recht 81

1. Der Vorlauf 1919-1924. Unterstützer und Teilhaber des Vaters 81

2. 1924-1933. Die ersten Jahre als Verleger 87

3. Heinrich Schönfelder 93

4. Der Greif 103

X. Das «Dritte Reich» und der Buchhandel. Rechtsverwüstung und Gleichschaltung 107 
XI. Heinrich Beck 1933-1945. Beginn von Größe im Juristischen und Nationalsozialistisches 111

1. Arisierungen 111

2. Der Verlag von Otto Liebmann 115

3. Der Erwerb des Verlags von Otto Liebmann 131

4. Der Verleger im «Dritten Reich» 138

5. Einzelne Werke 156

a) Hueck/Nipperdey/Dietz, Arbeitsorganisationsgesetz 156

b) Stuckart/Globke, Kommentar zur Rassegesetzgebung 159

c) Palandt, Bürgerliches Gesetzbuch 166

d) Volkmar/Antoni u. a., Großdeutsches Eherecht, Kommentar zum Ehegesetz vom 6. Juli 1938177

e) Schönke, Strafgesetzbuch 179

6. Andere Verlage mit Literatur zum Recht im «Dritten Reich» 180

XII. Westdeutsche Nachkriegszeit und ihr Buchhandel. 1945-1949 183

XIII. Entnazifizierungsverfahren gegen Heinrich Beck. JCS 1067, Militärregierungsgesetz Nr. 191189

XIV. Aufarbeitung von Unrecht des Nationalsozialismus. Rückerstattung. Entschädigung. 201

XV. Berliner Niederlassung und Nördlinger Druckerei nach Kriegsende 1945205

XVI. Biederstein Verlag. Statthalter für CHB 207

1. Gustav End 207

2. Der Anfang 1946/47 209

3. Gründung der Neuen Juristischen Wochenschrift 215

4. Die Wende 1948/49 217

XVII. Bauarbeiten in München und in der Nördlinger Druckerei. Ein vielseitig begabter Architekt: Roderich Fick 221

XVIII. Die Bundesrepublik und ihr Buchhandel. Unterwegs zum Wirtschaftswunder 229 
XIX. Heinrich Beck in der Bundesrepublik. 1949-1970.

C.H.Beck wird größter juristischer Verlag 233

1. C.H.Beck und Biederstein Verlag 233

2. Die Ära Höller 235

3. Neue Juristische Wochenschrift, Juristische Schulung und vier andere Zeitschriften 236

4. Textausgaben 245

5. Kommentare von Palandt bis zu Thomas/Putzo und Löffler 249

6. Kommentare zum Strafrecht. Grundlage für die Zukunft 255

7. Maunz/Dürig, Grundgesetz 262

8. Lehrbücher und Studienliteratur 271

a) Große Lehrbücher in der «Grünen Reihe» 272

b) Juristische Kurz-Lehrbücher 293

c) «Prüfe dein Wissen» und «Grundrisse des Rechts» 301

9. Rechtsgeschichte 303

XX. Hans Dieter Beck in der Bonner Republik. 1970-1990.

Expansion des Juristischen 309

1. Die sozialliberale Koalition und die Anfänge der Ära Kohl 309

2. Wechsel an der Verlagsspitze, Erwerb des Verlages Franz Vahlen und weitere Verlagsgebäude 311

3. Eine neue Klasse: der Münchener Kommentar zum Bürgerlichen Gesetzbuch 315

4. Die weitere Familie der Münchener Kommentare 321

a) Handelsgesetzbuch 321

b) Aktiengesetz 322

c) $\mathrm{GmbH}-$-Gesetz 324

d) Deutsches und Europäisches Wettbewerbsrecht 325

e) Insolvenzordnung 326

f) Versicherungsvertragsgesetz 326

g) Lauterkeits-, Bilanz- und Anfechtungsrecht 327

h) Zivilprozessordnung 327

5. Eine recht erfolgreiche Textausgabe und ein «Gelber Kommentar» zum BGB 328

6. Soziale Bewegung und andere Neuigkeiten im Privatrecht 330

a) Mietrecht 330

b) Arztrecht 332

c) Nichtehelichenrecht 333

d) Internationales Privatrecht 335 
7. Weitere Entwicklungen im Zivilrecht 339
a) Familienrecht 339
b) Erbrecht 342
c) Wohnungseigentumsrecht 343
d) Gesellschaftsrecht 348
e) Versicherungsrecht 349
f) Bankrecht 354
g) Wettbewerbs- und Immaterialgüterrecht 354
h) Kartellrecht 358
i) Vergaberecht 359
k) Privates Baurecht 361

8. Einige Bewegung im Prozessrecht 363

9. Handbücher sowie Vertrags- und Formularbücher 365
a) Sauter, Der eingetragene Verein 365
b) Geigel, Der Haftpflichtprozess 365
c) Handbuch der Rechtspraxis (HRP) 366
d) Bärmann/Seuß, Praxis des Wohnungseigentums 366
e) Gesellschaftsrechtliche Handbücher 366
f) Bankrechts-Handbuch 367
g) Weitere Handbücher 367
h) Münchener Vertragshandbuch 368
i) Beck'sches Formularbuch Bürgerliches, Handels- und Wirtschaftsrecht 369
k) Beck'sches Prozessformularbuch 370
I) Beck'sches Formularbuch für den Strafverteidiger 370
m) Disketten und CD-ROMs in Formularbüchern 371

10. Arbeitsrecht: Rechtsliteratur für ein uneinheitlich kodifiziertes Rechtsgebiet 371

11. Das Sozialgesetzbuch und die Folgen für das Verlagsprogramm 381

12. C.H.Beck als Gravitationszentrum des Verfassungsrechts 389

13. Das Verwaltungsrecht wird immer wichtiger 396

14. Zunehmende Bedeutung des Europarechts 401

15. Festigung der Verlagsstellung im Strafrecht 406

16. Straßenverkehrsrecht 410

17. Der Weg zum steuerrechtlichen Verlag 416

a) Erste Werke 416

b) Gesetzesnovellen im Steuerrecht 417

c) Gesetzestextausgaben und Veranlagungshandbücher 419 
d) «Deutsches Steuerrecht (DStR)» und weitere Zeitschriften 420

e) Steuerrecht bei Franz Vahlen 422

f) Steuerrechtskommentare in der Gelben Reihe 423

g) Die Blaue Reihe der «Beck'schen Steuerkommentare» 427

h) Wassermeyer, Doppelbesteuerung 430

i) Beck'scher Bilanz-Kommentar 430

k) Die Blaue Handbuchreihe 431

I) Internationales Steuerrecht 431

m)Beck'sches Steuerberater-Handbuch 432

n) Küttner, Personalbuch 433

o) Resümee 433

18. Die Studienliteratur wird lebendiger 433

a) Prüfe dein Wissen (PdW) 435

b) Kurz-Lehrbücher 436

c) JuS-Schriftenreine 438

d) Grundrisse des Rechts 439

e) Juristischer Studienkurs 442

f) Grundkurse 442

g) Lernbücher Jura 443

h) Juristische Fall-Lösungen 443

i) Studienkommentare 444

k) Beck'sche Examinatorien 444

I) Jurakompakt 444

m) Lehrbücher im Verlag Franz Vahlen 445

n) Lehrbücher von Heymanns, Luchterhand \& Co. im Verlag Franz Vahlen 445

19. Große Leistungen in der Rechtsgeschichte 448

20. «Neue» Zeitschriften entstehen in den achtziger Jahren und später fast eine Explosion 458

a) Die «N»-Zeitschriften 460

b) Weitere Zeitschriften-Neugründungen 464

c) Übernahmen von anderen Verlagen 464

d) Rechtsprechungs-Reporte (RR) 465

e) Zeitschriften bei Vahlen und Nomos 465

f) Jüngste Entwicklungen 465

\section{Deutschlands Vereinigung und Zerfall des Ostblocks.} Entstehung von Rechtsstaaten im östlichen Mitteleuropa 467 
XXII. Hans Dieter Beck in der Berliner Republik. 1990-2013. Die Expansion wird noch größer 471

1. Das Ende der DDR und die Folgen für den Verlag 471

2. Aufbruch ins östliche Mitteleuropa 476

a) Verlagsgründung in Warschau 476

b) Verlagsgründung in Prag 481

c) Der Verlag «All Beck» - Erwerb einer Beteiligung in

Rumänien und später eine Art Neugründung 484

3. Erwerb von Nomos und anderen Verlagen 486

4. «Beck International»- das englischsprachige Programm des Verlages 488

5. Neue oder grundlegend veränderte Rechtsgebiete 491

a) Informationstechnologie, Telekommunikation,

Datenschutz 491

b) Energierecht 497

c) Compliance 501

6. Neues auf dem Handbuchmarkt: Anwaltsbücher und weitere Formularbuchreihen 503

a) Handbücher speziell für den Anwalt 503

b) Formularbücher 507

7. Rote Kommentarreihe 509

8. Rechtsliteratur für Nichtjuristen 512

9. BeckAkademie Seminare 513

10. Die neue Form des Kommentars: der BeckOK (Beck'scher Online-Kommentar) 516

XXIII. beck-online - die Datenbank.

Publizieren in der digitalen Welt (von Hans Dieter Beck) 521

1. Rückblick auf die Entwicklung 521

2. Die neuere Entwicklung. Erreichter Stand 525

3. beck-online. Chancen und Risiken 529

4. Zur Zukunft der Bücher 532

XXIV. Die Beck'sche Unternehmensgruppe. Synergien (von Hans Dieter Beck) 535

1. Die Nördlinger Druckerei 535

2. Die Buchhandelsgruppe Schweitzer Fachinformationen 536

3. Auslandsverlage 538 
XXV. Dank und Ausblick (von Hans Dieter Beck) 541

Anhang 1: Kaufvertrag über den Erwerb des Verlages von Otto Liebmann vom 12. Dezember 1933545

Anhang 2: Spruchkammerentscheidung («Spruch») vom 1. Oktober 1947 betreffend das Entnazifizierungsverfahren gegen Dr. Heinrich Beck 551

Literaturhinweise 559

Abbildungsnachweis 573

Personenverzeichnis 575

Zur Person des Autors 591 
https://doi.org/10.17104/9783406684883-

Generiert durch IP '172.22.53.54', am 26.04.2023, 11:13:15.

Das Erstellen und Weitergeben von Kopien dieses PDFs ist nicht zulässig. 


\section{Veröffentlichungen des Verlags zu seiner Geschichte und Arbeit:}

Verlagskatalog der C.H. Beckschen Verlagsbuchhandlung Oscar Beck in München 1763-1913. Mit einer geschichtlichen Einleitung, 1913 (zitiert: Oscar Beck 1913)

Der Aquädukt. Ein Jahrbuch. Herausgegeben im 175.Jahr der C.H. Beck'schen Verlagsbuchhandlung, 1938 (zitiert: Heinrich Beck 1938)

Festschrift zum zweihundertjährigen Bestehen des Verlages Beck 17631963, 1963 (zitiert: Heinrich Beck 1963)

Der Aquädukt 1963. Im 2oo. Jahre ihres Bestehens herausgegeben von der C.H. Beck'schen Verlagsbuchhandlung, 1963

Drei Reden, Festgabe zum 8o. Geburtstag von Heinrich Beck, 1969 (zitiert: Heinrich Beck 1969)

Die C.H. Beck'sche Buchdruckerei Nördlingen in den Jahren 1945 bis 1979, 1980 (am Ende mit einer Stammtafel der Familie Beck, Auszug; zitiert: Albert Heinrich 1980)

Juristen im Porträt. Verlag und Autoren in 4, Jahrzehnten. Festschrift zum 225jährigen Jubiläum des Verlages C.H. Beck, 1988 (zitiert: Hans Dieter Beck 1988, sonst: Juristen im Portrait)

Der Aquädukt 1763-1988. Ein Almanach aus dem Verlag C.H. Beck, 1988 (zitiert: Wolfgang Beck 1988)

Einladung ins 18. Jahrhundert. Ein Almanach aus dem Verlag C.H.Beck, 1988

Bibliographie Verlag C.H.Beck 1913-1988. Biederstein Verlag 1946-1988. Verlag Franz Vahlen 1970-1988. Bearbeitet von Albert Heinrich, 1988 
Rechtswissenschaft und Rechtsliteratur im 2o. Jahrhundert. Mit Beiträgen zur Entwicklung des Verlages C.H.Beck, herausgegeben von Dieter Willoweit, 2007 (zitiert: Rechtswissenschaft und Rechtsliteratur)

Hermann Weber, Juristische Zeitschriften des Verlages C.H. Beck. Von den Anfängen im 19. Jahrhundert bis zum Zeitalter der elektronischen Medien, 2007

\section{Verlagsarchiv}

Das Archiv des Verlags ist am Ende des Zweiten Weltkriegs zerstört worden. Es gibt aber Unterlagen über den Erwerb des Verlags von Otto Liebmann, die nach dem Ende des Kriegs aus Berlin nach München gebracht worden sind (vgl. S. 131), und eine Abschrift des Kaufvertrags über diesen Verlag in der Akte des Staatsarchivs München zum Entnazifizierungsverfahren gegen Heinrich Beck, die auf S. 545 ff. wiedergegeben ist. 